\title{
Developments in AZtec: New Solutions for EBSD
}

\author{
J. Goulden ${ }^{1}$, A. Bewick ${ }^{1,}$ P. Trimby ${ }^{1}$ \\ 1. Oxford Instruments NanoAnalysis, Halifax Road, High Wycombe, UK
}

Electron backscatter diffraction EBSD is a powerful technique applicable to a range of microstructural analyses, including texture, grain size, boundary characterization, phase distribution and inter-phase orientation relationships. The technique is suited to solving an increasing range of analytical problems on the um to $\mathrm{mm}$ scale. However, best results are only obtained with a good understanding of how to optimise each element in a complicated analysis chain: from pattern acquisition and processing through to the final interpretation of the output data.

The Oxford Instruments AZtec platform is a state-of-the-art microanalysis system designed to ensure consistent optimization of EBSD experiments. AZtec embodies Oxford Instruments' expert understanding of the whole EBSD analysis system, enabling users to focus on their particular application and to worry less about the multitude of parameters that can affect the quality of their analyses. This presentation will outline some of the fundamental technologies lying behind an EBSD system and discuss how they impact (and potentially limit) the technique. Recent developments in hardware and software, resulting in an improved user experience and better analyses, will be discussed.

The basis of an EBSD system is a fast, sensitive and high dynamic range detector that captures the electron backscatter diffraction patterns (EBSPs). The detector is designed to operate over a wide range of conditions, but it must still be configured for optimal performance in each application. The sensitivity of the detector is most usefully discussed with reference to its signal-to-noise ratio (SNR) characteristic; the SNR varies as a function of electron dose as shown approximately in Figure 1. The electron dose is the total number of electrons used to generate the EBSD pattern, i.e. beam current $*$ exposure time. Typically, the electron dose is minimized (to reduce acquisition time and possible damage to the specimen) whilst still collecting EBSPs of sufficient quality (high enough SNR) for successful analysis. The minimum dose required depends not just on the detector's SNR, but also on the application in question. For example, high resolution-EBSD (HR-EBSD) requires a very high dose; materials with a weak diffraction signal require a relatively high dose for successful orientation mapping, whereas high-contrast EBSPs can be successfully collected at high speeds from many common metal samples using very low doses. The sensitivity of the EBSD detector, for a given application, is inversely related to the minimum dose required to achieve success with that detector in that application.

The ideal detector adds no noise to the EBSP, in which case the SNR is limited solely by Poisson counting statistics. Real detectors, however, do add noise (Figure 1 compares a slower, sensitive detector to a faster, less sensitive detector). This presentation will discuss the design parameters affecting how real detectors approach ideal SNR performance, how to choose a camera, and how AZtec helps the user to configure the system for optimum performance for specific applications.

In recent years, incremental detector developments have pushed the maximum frame rates of some EBSD detectors to in excess of 1500 patterns per second. However, high speed comes at a cost (to 
sensitivity, EBSP resolution and signal range), limiting the use of these detectors in a number of applications. Figure 2 shows approximate curves for the maximum possible successful analysis speed, for a given beam current. Two applications are illustrated: one $(1$ - solid lines $)$ with highcontrast EBSPs (e.g. from undeformed semiconductors or metals) where state-of-the-art sensitive and fast EBSD detectors may achieve sensitivities of approximately $500 \mathrm{~Hz} / \mathrm{nA}$ and $140 \mathrm{~Hz} / \mathrm{nA}$ respectively, and a second ( 2 - dashed lines) for samples generating lower-contrast EBSPs, where sensitivities may be much lower. An ideal detector would combine fast speed and high sensitivity as denoted by the blue curves in Figure 2.

The sensitive detector performs very well at low current but has a very limited maximum speed. The fast detector requires a higher current for a given speed, with a much higher maximum speed. This high speed limit may be achievable on materials with strong diffraction, if the required beam current is available. The higher dose requirement for weaker diffracting materials also requires less binning of the detector's CCD image sensor to avoid saturating the pixels; the maximum speed is thus reduced. Optimizing the configuration to different applications will be discussed.

Pattern processing also influences the sensitivity and speed of EBSD analysis. Typically, EBSD pattern processing involves the application of one or more background corrections which are intended to remove all contrast in the patterns, other than that related to diffraction. This will 'flatten' the steep profile of the raw EBSD pattern (bright centre and dark edges), and also correct for phosphor screen defects (e.g. scratches). Modern EBSD systems will typically offer 'static' and 'dynamic' backgrounds. A static background correction requires the collection of a suitable reference image, whilst the dynamic (or 'auto') requires only the working EBSP. Each method has its advantages and disadvantages. Innovations that simplify the process of applying background correction whilst also making them more robust across all applications will be introduced. These greatly enhance the ease of use and efficiency of EBSD work.

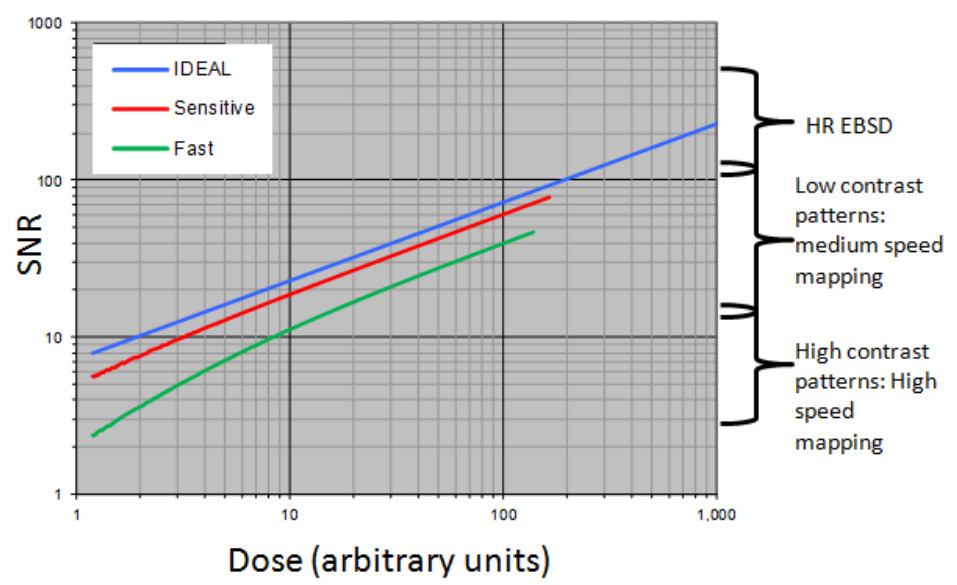

Figure 1. Comparison of SNR for different detectors (single frame), also showing typical application space

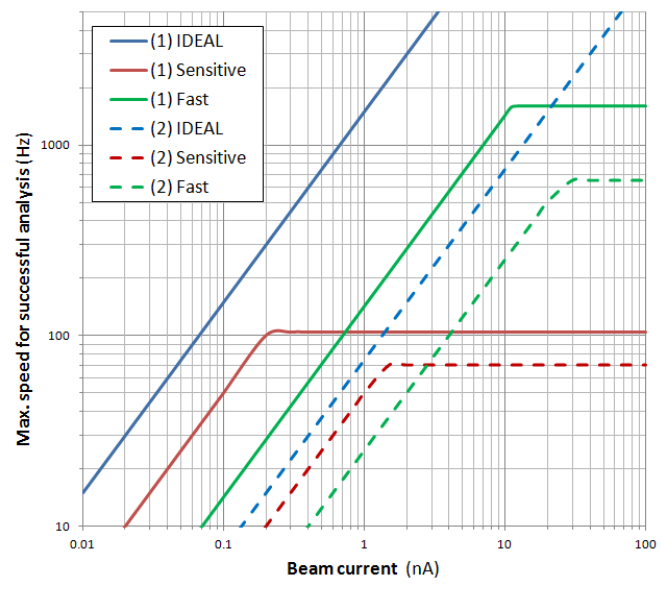

Figure 2. Detector performance showing maximum possible successful analysis speed, for a given beam current for two different applications (1) and (2) 